\title{
Associations between testosterone secretion and sexual activity in women
}

\author{
Sari M. van Anders *, Lisa Dawn Hamilton, Nicole Schmidt, Neil V. Watson \\ Department of Psychology, Simon Fraser University, 8888 University Drive, Burnaby BC, Canada V5A 1 S6
}

Received 1 November 2006; revised 15 January 2007; accepted 16 January 2007

Available online 25 January 2007

\begin{abstract}
Some studies show an increase in testosterone (T) after sexual activity; this literature has inconsistent findings, focuses mostly on men, and does not employ control activities. The present study examined within-subject effects of intercourse versus control activities (cuddling; exercise) on salivary $\mathrm{T}$. The initial sample included 49 women (mostly heterosexual), though not all participants returned all samples or engaged in all activities, leaving a smaller sample for endocrine analyses $(n=16)$. Participants attended an initial session in the laboratory where they completed questionnaires, and then engaged in the activities on their own. On three separate nights, they provided pre-activity, post-activity, and nextmorning saliva samples and completed brief questionnaires at the last two timepoints. Women's $\mathrm{T}$ was higher pre-intercourse than pre-control activity. Women's $\mathrm{T}$ was also higher post-intercourse than post-control activity, though the percent change in $\mathrm{T}$ from pre- to post-activity was highest for cuddling, then intercourse, then exercise. Next-morning $\mathrm{T}$ did not differ by activity. Data pointed to an association between $\mathrm{T}$ and orgasming, sexual desire, and relationship commitment. Analyses on post-activity appraisals suggest that the close intimate physicality of a sexual and non-sexual nature can affect $\mathrm{T}$ and be beneficial in short-term and perhaps longer-lasting ways for women's sexuality and relationships. (C) 2007 Elsevier Inc. All rights reserved.
\end{abstract}

Keywords: Sexual activity; Androgen; Women; Orgasm; Sexual desire; Exercise; Cuddling; Intercourse; Testosterone

In many species, androgens play a critical role in sexual activity (for a review, see Nelson, 2000). Prenatal exposure to androgens can influence sexual behavior in adulthood, and the reverse relationship is also observed: engaging in sexual behaviors can alter circulating androgen concentrations. The majority of human research examining the effects of sexual behaviors on androgens has focused on the androgenic response of heterosexual men to vaginal intercourse and masturbation or viewing of erotic films (for a review, see van Anders and Watson, 2006b).

Viewing sexually explicit movies appears to increase testosterone ( $\mathrm{T}$ ) levels compared to viewing neutral films in men (sample sizes ranging from 7 to 20: Hellhammer et al., 1985; Pirke et al., 1974; Rowland et al., 1987; Stoleru et al., 1993; cf. Carani et al., 1990), but perhaps not in women (Heiman et al., 1991) though these analyses did not compare pre- and post-exposure T levels. Similarly, engaging in sexual activity generally appears to produce an increase in $\mathrm{T}$ titers,

\footnotetext{
* Corresponding author. Fax: +1 6042913427.

E-mail address: saria@sfu.ca (S.M. van Anders).
}

though not as consistently. Masturbation leads to increases in $\mathrm{T}$ relative to pre-masturbation $\mathrm{T}$ in men ( $n=34$, Purvis et al., 1976; cf. Krüger et al., 1998) and women ( $n=10$, Exton et al., 1999). Although several studies have failed to find this association between intercourse and an increase in $T$, these are based on notably small sample sizes (e.g. one man: Fox et al., 1972; eight men, five women: Lee et al., 1974; six men: Stearns et al., 1973). Despite also having a small sample Dabbs and Mohammed (1992) found the expected increase in $\mathrm{T}$ in a sample of four heterosexual couples. The time course between engaging in sexual activities and sampling $\mathrm{T}$ (from blood or saliva) may be a factor in some studies; for example, Kraemer et al. (1976) observed a T increase in a sample of 19 men only when measured $24 \mathrm{~h}$ after intercourse. Similarly, men engaging in sexual activity with unfamiliar or multiple partners showed higher $\mathrm{T}$ the following morning (Hirschenhauser et al., 2002).

A major limitation of past studies is their lack of appropriate control activities since partnered sexual activity involves physical activity, physical arousal (e.g. increased heart rate from movement), sexual arousal, and physical closeness/ 
intimacy with a partner. The lack of control activities makes it unclear whether increases in $\mathrm{T}$ following sexual activity are due to the sexual nature of these activities or other influences like arousal (e.g. increased heart rate) or physical contact (e.g. skinto-skin). In the present study, we attempted to examine how sexual activity resulted in increased $\mathrm{T}$ compared to two control activities: (1) exercise, which we chose to control for the physical arousal aspect of sexual activity, and (2) cuddling, which we chose to control for the close physical intimacy. Previous studies have rarely included women, and the three studies that did so included only small samples (4-10 women; Dabbs and Mohammed, 1992; Exton et al., 1999; Lee et al., 1974). In the present study, we examined women and attempted to recruit larger samples. In addition, we asked participants to engage in activities in their own homes to avoid an artificial and sterile laboratory environment. Finally, we sampled at two postactivity timepoints to capture any immediate and/or longerlasting changes in $\mathrm{T}$ and to compare changes in $\mathrm{T}$. We were interested in seeing whether sexual activity affected $\mathrm{T}$ differently than control activities, how $\mathrm{T}$ was associated with other sexual parameters like orgasms and sexual desire, and how evaluations of the activities might differ depending on their sexual or intimate nature.

\section{Methods}

\section{Participants}

Participants were recruited through posters at Simon Fraser University (SFU), the University of British Columbia, and the Greater Vancouver Regional District, as well as through the SFU Psychology Participant Pool. Recruitment was aimed at healthy, sexually active people with a regular sexual partner. This research was approved by the SFU Research Ethics Board.

All participants were aware of the sexual nature of the study before they contacted the experimenter for more information. Before participating, they read and signed an Informed Consent Form that noted that sexual activity carries risks of sexually transmitted infections and that these can be reduced using safer-sex practices (some of which were listed). Participants were informed that they would not be asked to engage in sexual activities outside of their usual pattern of sexual activity, leading to no extra risk from participating in the study.

There were 49 women (mean age $=21.33$ years, $S D=3.80$ years) participating in the study (note that not all women returned all samples; see Results for numbers by analyses), 29 of whom were using hormonal contraceptives (HC). Though HCs alter endogenous hormone production and are associated with lower T parameters (van Anders and Watson, 2006a), HC use was not a criterion for exclusion because we were interested in individual changes from baseline; each participant was thus her own control. Participants self-identified their sexual orientation: the majority self-identified as heterosexual $(n=44)$, three women identified as bisexual, one as lesbian, and one as queer. Participants were mostly students $(n=43)$ though many worked at other part-time jobs. Occupations included: clerical work, customer research analysis, hostess, mental health worker, model, office assistant, residential care worker, retail worker, waitress, and writer. One participant had children. Participants were diverse ethnically. ${ }^{1}$

\footnotetext{
${ }^{1}$ Caucasian or White or European $(n=21)$, Chinese $(n=9)$, Asian or AsianCanadian $(n=6)$, Hispanic $(n=4)$, and Portuguese or Portuguese-Canadian $(n=2)$. One participant in each of the following: Canadian, East Indian, German-Métis, Indian, n/a, Native-Norwegian, Persian, South African-East Indian, and White-Aboriginal.
}

\section{Procedure}

Participants attended a laboratory session at which they completed consent forms, health and background questionnaires, the Profile of Mood States (McNair et al., 1971), and the Sexual Desire Inventory (Spector et al., 1996). They then provided a saliva sample to practice the process and facilitate home collection (we did not analyze this sample). Participants were given a take-home package, which included an Activity Sheet assigning intercourse and control activities (cuddling; exercise). The order of the activities differed by participant such that participants were randomly assigned to begin with each activity. At closing, participants were offered information on safer-sex and contraception.

Participants engaged in one activity per night, on three nights spread generally over 1 week. Participants provided three saliva samples associated with each activity. Sample 1 (pre-activity) was taken just before dinner; this provided an opportunity for rough standardization to a common event (dinner) and to avoid food contamination. Participants were instructed to engage in the activities at some point after dinner in the evening. At $15 \mathrm{~min}$ post-activity, participants provided Saliva Sample 2 (post-activity) and completed the Postactivity Questionnaire. Having participants engage in the activities at night allowed us to reduce the diurnal variance associated with $\mathrm{T}$ and sleep, especially since levels are more stable in the evening (Axelsson et al., 2005). The morning following an activity, participants provided Saliva Sample 3 (next-morning sample) and completed the Next Morning Questionnaire. This was done before breakfast to avoid food contamination and provide a rough standardization to another common event (breakfast).

\section{Materials}

\section{Questionnaire}

We asked participants to respond to questions about health, demographics, relationships (including commitment), and sexuality. Participants were also asked whether they were comfortable having sexual activities assigned for study's purposes after being informed that they could choose their own sexual activities or have them assigned. Participants also indicated the frequency of partnered and solitary sexual activity and orgasms, and the frequency of orgasms relative to sexual activity. Participants also indicated their degree of enjoyment and their frequency of engaging in a list of various sexual activities we provided.

Participants completed the Sexual Desire Inventory (SDI: Spector et al., 1996) for assessment of levels of sexual desire, modified slightly by the addition of one question "During the last month, how often have you had sexual thoughts?" (see van Anders et al., 2005). The SDI produces a Total SDI score, a Solitary SDI score, and a Dyadic SDI score.

\section{Take-home package}

This contained polypropylene tubes, tube lids, parafilm squares, gum (Trident sugar-free cherry; previously shown to be inert in past studies: Dabbs, 1991), an indelible marker, a pen, an instruction sheet, and a record sheet. It also contained two envelopes containing questionnaires. The first was the 'Post-activity Questionnaire', in which participants indicated the activity, date, and time. Participants also indicated whether they orgasmed with their activity (yes/no), level of intimacy with partner (five-point scale from ${ }^{\prime} 0$ ' $=$ 'not at all' to ' 5 ' =' 'extremely'), enjoyment (same scale), and sexual pleasure (same scale). We included enjoyment and sexual pleasure because activities can be enjoyed, e.g. exercise, without being sexually pleasurable. The second was the 'Next Morning Questionnaire', in which participants indicated the activity from the night before, date, time, energy, interest in sexual activity, feelings of sexual attractiveness, and level of intimacy (all on the same scale as above).

\section{Saliva samples and hormone measurement}

As salivary measures of steroid hormones represent the bioavailable or weakly bound portion (Quissell, 1993), they are an appropriate measure for assessing changes in T. And, it is this portion that is hypothesized to be able to travel to and bind with neural receptors, so it is an appropriate measure for psychological studies. Testosterone levels in saliva assays correlate highly with participants' free testosterone concentration in serum (Wang et al., 1981). While 
salivary measures of T are reliable and accurate (Granger et al., 2004), they tend to underestimate behavior-testosterone relationships in women compared to serum samples (Granger et al., 2004). Because of this, results that show salivary testosterone-behavior relationships in women are not undermined, but null findings in women may mask underlying relationships. Despite this and because salivary $\mathrm{T}$ also provides the benefits of a non-invasive, easily repeatable, selfadministered sampling method - all of which were vital methodological concerns for this study - salivary samples were used.

Saliva was stimulated with the use of an inert gum (Trident cherry sugarfree), and participants were requested to refrain from eating, smoking, brushing their teeth, or drinking beverages other than water for $1 \mathrm{~h}$ prior to testing. Saliva samples were collected in polypropylene tubes that had been pretreated with sodium azide. Samples were frozen after collection at $-20^{\circ} \mathrm{C}$ and assayed for testosterone in duplicate using a modified kit from Diagnostic Systems Laboratories (Webster, TX) at the Core Biomarkers Laboratory at Yerkes National Primate Research Center at Emory University. The assay range was $2-500 \mathrm{pg} / \mathrm{mL}$ at a $200 \mu \mathrm{g}$ dose. The interassay coefficient of variance was $8.77 \%$ at $0.65 \mathrm{ng} / \mathrm{mL}, n=14$ and $6.88 \%$ at $5.06 \mathrm{ng} / \mathrm{mL}, n=14$. The intraassay coefficient of variance was $6.54 \%$ at $98.82 \mathrm{pg} / \mathrm{mL}, n=8$.

\section{Analyses}

Analyses were conducted using the Statistical Package for the Social Sciences (SPSS) version 13.0. Some of the women's T levels were below assay sensitivity levels $(2 \mathrm{pg} / \mathrm{mL})$; these were entered as $1 \mathrm{pg} / \mathrm{mL}$ to reflect low concentrations and avoid data loss. Analyses were conducted using repeated measures ANOVAs (analyses of variance), and following significant overall effects or trends, post hoc analyses were conducted using the Least Significant Differences (LSD) tests. Correlations were Pearson Product Moment Correlations. Differences between two means were conducted using independent $t$-tests, when appropriate.

\section{Results}

To check that the diurnal rhythm in $\mathrm{T}$ (higher $\mathrm{T}$ in the morning upon waking, Axelsson et al., 2005) was apparent in our data, we conducted a repeated measures analysis of variance (ANOVA) with time (pre-activity, post-activity, next-morning) and three contexts (exercise, intercourse, cuddling). The overall effect of time was significant for exercise, $F(2,28)=14.68, p<$ .01 , intercourse, $F(2,28)=5.94, p<.01$, and cuddling, $F(2,28)=$ $8.79, p<.01$, with highest levels in the morning.

Normal female ranges for morning and evening salivary $\mathrm{T}$ vary per laboratory and methodology. As such, the expected range for women's $\mathrm{T}$ can be as low as undetectable to about $45 \mathrm{pg} / \mathrm{mL}$ relative to past studies with assays conducted by the same laboratory. One previous study using similar methodology reported means of $22.09 \mathrm{pg} / \mathrm{mL}$ at 08:00, $17.10 \mathrm{pg} / \mathrm{mL}$ at 09:30, and 14.42 pg/mL at 19:00-21:00 (van Anders and Hampson, 2005). There are seasonal (van Anders et al., 2006) and other effects on $\mathrm{T}$, so it is not accepted practice to use other studies as standards. Still, values in the present study were comparable to published values.

Analyses contrasting within-subject differences in $\mathrm{T}$ by activity were limited to women who engaged in all the activities of interest so that repeated measures analyses could be carried out, leaving 17 self-identified heterosexual women for statistical analyses. There was one missed sample for the post-activity and morning timepoints, so those analyses were on 16 women. For the purposes of analyses between $\mathrm{T}$ and orgasms and sexual desire, 28 women who returned intercourse samples were included in analyses. For analyses not including $\mathrm{T}$ samples, the original 49 women were included.

\section{Effects of sexual vs. control activities on testosterone (T)}

There was a significant overall effect of the three activities on pre-activity T, $F(2,32)=6.07, p<.01$. Pre-intercourse T was significantly higher than pre-cuddling $\mathrm{T}, p<.01$, and preexercise $\mathrm{T}, p=.05$. $\mathrm{T}$ did not differ significantly between precuddling and pre-exercise, $p=.22$ (see Fig. 1). Thus, T appears to be higher prior to intercourse compared to cuddling and exercise.

There was a significant overall effect of the three activities on post-activity $\mathrm{T}, F(2,22)=5.50, p=.02$ (see Fig. 1 for means). Post-intercourse $\mathrm{T}$ was significantly higher than post-exercise $\mathrm{T}$, $p=.015$, and nearly so compared to post-cuddling $\mathrm{T}, p=.061$. T did not differ significantly between post-cuddling and postexercise, $p=.12$. Post-activity levels could reflect pre-activity levels of $\mathrm{T}$, which did differ by activity. To check this and because we were interested in changes from pre- to postactivity, we calculated an additional $\mathrm{T}$ variable (percent change in $\mathrm{T}$ ) by subtracting participants' pre-activity $\mathrm{T}$ from postactivity $\mathrm{T}$ and dividing this change in $\mathrm{T}$ by pre-activity $\mathrm{T}$ (and then multiplying by 100). This provided a measure of the change in $\mathrm{T}$ referenced to the starting level of $\mathrm{T}$. We then conducted a repeated measures ANOVA with the three activities using the percent change in $\mathrm{T}$. There was a trend for a significant overall effect, $F(2,30)=2.65, p=.09$, so we continued with post hoc tests (see Fig. 1 for means). The percent change in $\mathrm{T}$ for intercourse approached significance to be higher than the percent change in $\mathrm{T}$ for exercise, $p=.072$, and was not significantly different from the percent change in $\mathrm{T}$ for cuddling, $p=.52$. The percent change in $\mathrm{T}$ for exercise was significantly lower than the percent change in $\mathrm{T}$ for cuddling, $p=.05$. Thus, $\mathrm{T}$ is higher following intercourse compared to cuddling or exercise. This increase in $\mathrm{T}$ from pre- to postactivity appears to be higher for intercourse and cuddling compared to exercise.

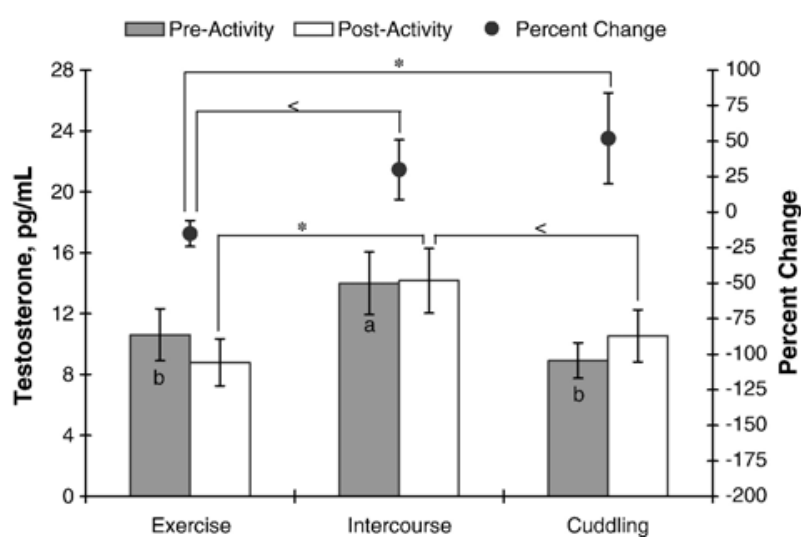

Fig. 1. Mean pre-activity, post-activity, and percent change in testosterone levels by activity with standard error bars '*' and differing subscripts indicate significant differences at $p<.05$. ' $<$ ' Indicates a trend towards a significant difference at $p<.10$. 
There was no evidence for a significant overall effect of the three activities on next-morning T, $F(2,30)=1.71, p=.21$.

\section{Orgasms}

We divided women engaging in intercourse into orgasm $(n=18)$ and no-orgasm $(n=10)$ groups to see if the T measures associated with intercourse were related to orgasming. There was one outlier in the no orgasm group (over $3 \mathrm{SD}$ from the mean for pre- and post-intercourse T, and just under 3 SD for next-morning $\mathrm{T}$ ) who we excluded from this analysis. We conducted a repeated measures ANOVA, and women who orgasmed had significantly higher average $\mathrm{T}$ than women who did not $F(1,24)=4.74, p=.04$ (see Fig. 2a for means). Orgasming did not appear to lead to increased $\mathrm{T}$ as the percent change in $\mathrm{T}$ for women who orgasmed and women who did not orgasm did not differ significantly, $t(26)=-.24, p=.81$ (see Fig. $2 b$ for means).

Because the above suggested a trait association between orgasms and $\mathrm{T}$, we conducted correlations between reported orgasmic frequency and T. Pre-intercourse T was significantly correlated with reported frequency of orgasm when engaging in sexual activity, $r(26)=.48, p=.01$, and there was a trend for a correlation with post-activity $\mathrm{T}, r(26)=.31, p=.10$. Reported frequency of solitary orgasms was significantly correlated with morning after intercourse $\mathrm{T}, r(25)=.62, p<.01$, but not with pre-intercourse or post-intercourse $\mathrm{T}$. There were no significant

a

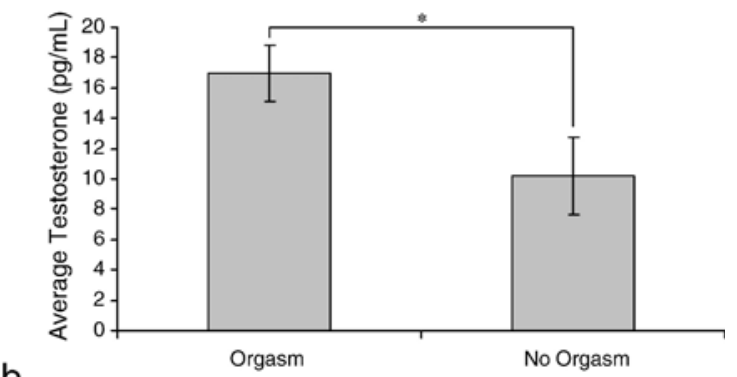

b

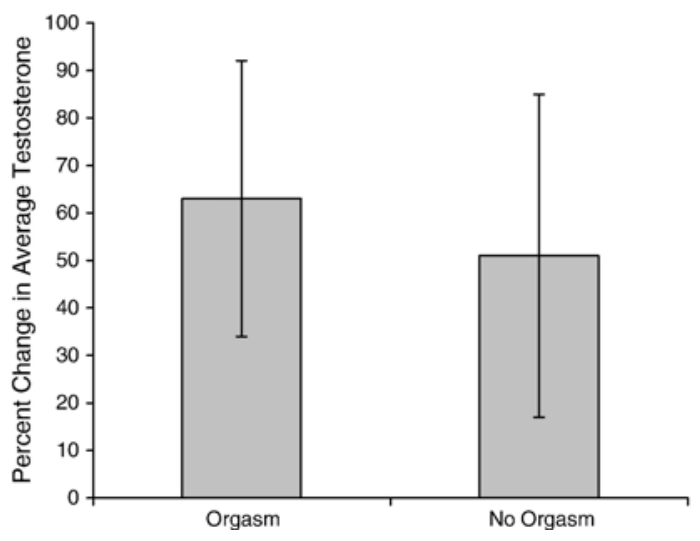

Fig. 2. (a) Mean average intercourse-related testosterone levels with standard error bars by presence of orgasm after intercourse. (b) Mean percent change in average intercourse-related testosterone from pre- to post-activity with standard error bars. '*' Indicates a significant difference at $p<.05$. correlations between women's reported frequency of partnered orgasms and any of the T measures.

To see if orgasmic frequency and relationship commitment were associated, we conducted correlations. Relationship commitment was significantly negatively correlated with solitary orgasms, $r(47)=-.49, p<.001$, and solitary sexual activity, $r(47)=-.51, p<.001$, but was not significantly correlated with partnered orgasms or partnered sexual activity. Relationship commitment was significantly negatively correlated with nextmorning $\mathrm{T}, r(25)=-.39, p=.044$, but not pre- or post-activity $\mathrm{T}$.

Thus women with higher $\mathrm{T}$ appear to be more likely to have orgasms and to report more solitary orgasms and less commitment. As well, relationship commitment appears to be negatively correlated with solitary sexual activity and orgasms.

\section{Sexual desire inventory}

Morning after intercourse T was significantly and positively correlated with SDI Total, $r(26)=.42, p=.034$, and SDI Solitary, $r(26)=.49, p=.012$, but not SDI Dyadic, $r(26)=.19, p=.357$. Neither pre-activity nor post-activity $\mathrm{T}$ showed any significant correlations with sexual desire. Thus, women's next-morning T appears to be associated with their sexual desire, especially solitary sexual desire.

\section{Post-activity evaluations}

After each activity, participants reported their sexual pleasure, enjoyment, and intimacy. There was a significant overall difference between the activities for sexual pleasure, $F(2,36)=55.38, p<.01$, and intimacy, $F(2,36)=7.66, p<.01$, and a trend for significant overall effect for enjoyment, $F(2,36)=2.85, p=.07$. Post hoc analyses (LSD) of simple main effects show the following significant results (all $p$ 's $<.05$ ). Sexual pleasure was significantly higher following intercourse than cuddling, which was significantly higher than exercise. Enjoyment was significantly higher following intercourse than exercise, but not cuddling. Intimacy was significantly higher following intercourse and cuddling than exercise. Thus, women reported more sexual pleasure following intercourse than control activities, and more enjoyment and intimacy following intercourse than exercise. Women also reported more sexual pleasure and intimacy following cuddling than exercise.

\section{Morning evaluations}

Similarly, the morning after each activity, participants reported their energy, interest in sexual activity, feelings of sexual attractiveness, and intimacy. There was a significant overall difference between the activities for feelings of sexual attractiveness, $F(2,36)=4.89, p=.01$, and intimacy, $F(2,36)=$ $3.23, p=.05$, but not for energy, $F(2,36)=.54, p=.59$, or interest in sexual activity, $F(2,36)=1.82, p=.18$. Post hoc analyses (LSD) show the following significant results (all $p$ 's $<.05$ ). Participants reported feeling significantly more sexually attractive the morning following intercourse compared to exercise or cuddling. Intimacy was significantly higher the 
morning following intercourse compared to exercise, but not cuddling.

\section{Discussion}

In the present study, we examined how sexual activities relative to control activities might affect testosterone (T) in women. We found that $\mathrm{T}$ was higher prior to intercourse compared to cuddling or exercise in women, suggesting anticipatory effects of sexual activity on androgens in women. We also found that $\mathrm{T}$ was higher $15 \mathrm{~min}$ after intercourse compared to exercise or cuddling and that the percent change in $\mathrm{T}$ from pre- to post-activity was higher for cuddling and intercourse than exercise. This suggests that increases in women's $\mathrm{T}$ might be mediated in part by close physical contact and/or intimacy. We were interested in next-morning $\mathrm{T}$ levels because this timepoint may be more telling of long-lasting effects, but there was no evidence that next-morning $\mathrm{T}$ levels differed depending on the previous night's activity. Our finding of an immediate increase in women's T after intercourse and no effect of evening intercourse on women's $\mathrm{T}$ the following morning contrasts with findings from men (e.g. Lee et al., 1974) where intercourse generally only appears to lead to higher $\mathrm{T}$ after a longer duration has elapsed, e.g. the next morning (e.g. Kraemer et al., 1976).

Our results suggest that individuals may be able to affect their own $\mathrm{T}$ by engaging in intercourse and, possibly, cuddling. Why is $\mathrm{T}$ higher prior to intercourse than control activities, and why does $\mathrm{T}$ increase closely afterwards intercourse and cuddling compared to pre-activity levels? One possibility relates to the reinforcing properties of androgens (Wood, 2004); increased $\mathrm{T}$ might be adaptive in reinforcing sexual activity, and it remains to be seen whether pre- or post-activity increases in $\mathrm{T}$ predict future sexual activity. Furthermore, androgen receptors (AR) in vaginal and clitoral tissue may be important in genital sexual arousal and function (in rabbits: Traish et al., 2002) and are widely dispersed throughout the central nervous system (CNS), so increased T may upregulate AR (Sader et al., 2005) and have positive effects on sexuality via the genitals or the CNS. The pre-intercourse increase in T could affect sexual sensations or perceptions directly through the genitals or the CNS, and previous studies have found that exogenous $\mathrm{T}$ coupled with erotic stimuli does increase arousal and lust (Tuiten et al., 2000).

Additionally, we found that women's T was associated with their sexual desire (total and solitary, but not dyadic), and our study appears to be the first to report that healthy women with higher $\mathrm{T}$ report more orgasms; these findings lead to the possibility that increased $\mathrm{T}$ from sexual activity might lead to increases in sexual desire or orgasmic experience. The significant association between $\mathrm{T}$ and sexual desire in women contrasts with previous research finding no association in healthy women (van Anders and Hampson, 2005), and further research is obviously required to clarify this relationship.

Notably, the number of women's solitary - but not partnered orgasms was associated with $T$, even though their $T$ was positively correlated with how often they experienced orgasms when they engaged in sexual activity. This pattern is similar to sexual desire in that solitary but not partnered sexual desire was associated with T. Additionally, women's report of relationship commitment and solitary orgasms was negatively correlated. The analysis of women's solitary and partnered orgasms is new to biobehavioral research, but is obviously a meaningful distinction that future studies should employ to help us understand how and why solitary and partnered orgasms are differentially associated with endocrine and relationship parameters.

We found that women felt more sexual pleasure, enjoyment, and intimacy immediately following intercourse compared to exercise and sometimes cuddling, but also more sexual pleasure and intimacy following cuddling than exercise. This suggests that the higher post-activity $\mathrm{T}$ following intercourse and cuddling may be associated with both sexual pleasure and intimacy, but not physical activity or energy expenditure per se. As such, the close physical intimacy and contact from both sexual activity and cuddling appear to have positive effects on intimacy and pleasure in a way that sharing time in other ways (e.g. exercise) does not. Interestingly, participants felt more sexually attractive the morning following intercourse compared to control activities, and more intimate the morning following cuddling and intercourse compared to exercise. This suggests that intercourse and cuddling may have positive effects that are apparent potentially the morning afterwards on important parameters of relationship quality and sexuality.

There are limitations in the present study, including social desirability affecting participants' responses (e.g. presence of orgasm, mood descriptors). As well, participants volunteering for studies involving sexual activities may not be representative of the larger population, though in studies of physiological reactions this is arguably of a lesser concern than in studies of attitudes and desires. Our use of the home environment with no experimenters present, and saliva samples taken outside the actual sexual activity, also probably led to a more realistic testing situation. One possibly confounding issue is that we used $\mathrm{T}$ measures from around intercourse to correlate with general sexuality measures, though intercourse was generally associated with increased $\mathrm{T}$ in our study. Thus, future studies should collect baseline $\mathrm{T}$ samples to correlate with trait measures of sexuality like desire and orgasm frequency. Additionally, studies including additional sexual activities and non-heterosexual women would provide important information.

Our research suggests that intercourse is associated with higher $\mathrm{T}$ in women before intercourse and that intercourse and cuddling increase $\mathrm{T}$ in women closely afterwards. This does not appear to be mediated by the physical activity component of sexual activity as exercise never led to increased T. Instead, it appears that sexual contact and close physical intimacy mediate the increased $\mathrm{T}$ as $\mathrm{T}$ was higher closely following cuddling as well. Findings point to a possible trait association between $T$ and orgasming. Our data also suggest that the close intimate physicality of a sexual and non-sexual nature (i.e. cuddling) can be beneficial in short-term and perhaps longer-lasting ways for women's sexuality and relationships. 


\section{Acknowledgments}

This research was supported by a Social Sciences and Humanities Research Council Institutional Grant-Small from Simon Fraser University. S.M. van Anders was supported by scholarships from the Natural Sciences and Engineering Research Council and IODE Canada. Salivary assays were conducted by the Core Biomarkers Laboratory at Yerkes Primate Research Center, Emory University. We would like to thank J. Bancroft, K. Zucker, and three anonymous reviewers for their comments on earlier drafts.

\section{References}

Axelsson, J., Ingre, M., Akerstedt, T., Holmback, U., 2005. Effects of acute displaced sleep on testosterone. J. Clin. Endocrinol. Metab. 90, 4530-4535.

Carani, C.J., Bancroft, J., Del Rio, G., Granata, A.R.M., Facchinetti, F., Marrama, P., 1990. The endocrine effects of visual erotic stimuli in normal men. Psychoneuroendocrinology 15, 207-216.

Dabbs Jr., J.M., 1991. Salivary testosterone measurements: collecting, storing, and mailing samples. Physiol. Behav. 49, 815-817.

Dabbs Jr., J.M., Mohammed, S., 1992. Male and female salivary testosterone concentrations before and after sexual activity. Physiol. Behav. 52, 195-197.

Exton, M.S., Bindert, A., Kruger, T., Scheller, F., Harmann, U., Schedlowski, M., 1999. Cardiovascular and endocrine alterations after masturbationinduced orgasm in women. Psychosom. Med. 61, 280-289.

Fox, C.A., Ismail, A.A.A., Love, D.N., Kirkham, K.E., Loraine, J.A., 1972. Studies on the relationship between plasma testosterone levels and human sexual activity. J. Endocrinol. 52, 51-58

Granger, D.A., Shirtcliff, E.A., Booth, A., Kivlighan, K.T., Schwartz, E.B., 2004. The "trouble" with testosterone. Psychoneuroendocrinology 29, 1229-1240.

Heiman, J.L., Rowland, D.L., Hatch, J.P., Gladue, B.A., 1991. Psychophysiological and endocrine responses to sexual arousal in women. Arch. Sex. Behav. 20, 171-186.

Hellhammer, D.H., Hubert, W., Schürmeyer, T., 1985. Changes in saliva testosterone after psychological stimulation in men. Psychoneuroendocrinology $10,77-81$.

Hirschenhauser, K., Frigerio, D., Grammer, K., Magnusson, M.S., 2002. Monthly patterns of testosterone and behavior in prospective fathers. Horm. Behav. 42, 172-181.

Kraemer, H.C., Becker, H.B., Brodie, H.K.H., Doering, C.H., Moos, R.H., Hamburg, D.A., 1976. Orgasmic frequency and plasma testosterone levels in normal human males. Arch. Sex. Behav. 5, 125-132.

Krüger, T., Exton, M.S., Pawlak, C., von zur Muhlen, A., Hartmann, U., Shedlowski, M., 1998. Neuroendocrine and cardiovascular response to sexual arousal and orgasm in men. Psychoneuroendocrinology 23, 401-411.

Lee, P.A., Jaffe, R.B., Midgley Jr., A.R., 1974. Lack of alteration of serum gonadotropins in men and women following sexual intercourse. Am. J. Obstet. Gynecol. 120, 985-987.
McNair, D.M., Lorr, M., Droppleman, L.F., 1971. Profile of Mood States. EdITS, San Diego.

Nelson, R.J., 2000. An Introduction to Behavioral Endocrinology, 2nd ed Sinauer Associates, Inc., Sunderland, Massachusetts.

Pirke, K.M., Kockott, G., Dittmar, F., 1974. Psychosexual stimulation and plasma testosterone in man. Arch. Sex. Behav. 3, 577-584.

Purvis, K., Landgren, B.-M., Cekan, Z., Diczfalusy, E., 1976. Endocrine effects of masturbation in men. J. Endocrinol. 70, 439-444.

Quissell, D.O., 1993. Steroid hormone analysis in human saliva. Ann. N.Y. Acad. Sci. 694, 143-145.

Rowland, D.L., Heiman, J.R., Gladue, B.A., Hatch, J.P., Doering, C.H., Weiler, S.J., 1987. Endocrine, psychological and genital response to sexual arousal in men. Psychoneuroendocrinology 12, 149-158.

Sader, M., McGrath, K., Hill, M., Bradstock, K., Jiminez, M., Handelsman, D., Celermajer, D., Death, A., 2005. Androgen receptor gene expression in leucocytes is hormonally regulated. Implications for gender differences in disease pathogenesis. Clin. Endocrinol. 62, 56-63.

Spector, I.P., Carey, M.P., Steinberg, L., 1996. The sexual desire inventory: development, factor structure, and evidence of reliability. J. Sex. Marital. Ther. 22, 175-190.

Stearns, E.L., Winter, J.S.D., Faiman, C., 1973. Effects of coitus on gonadotropin, prolactin and sex steroid levels in man. J. Clin. Endocrinol. Metab. 37, 687-690.

Stoleru, S.G., Ennaji, A., Cournot, A., Spira, A., 1993. LH pulsatile secretion and testosterone blood levels are influenced by sexual arousal in human males. Psychoneuroendocrinology 18, 205-218.

Traish, A.M., Kim, N., Min, K., Munarriz, R., Goldstein, I., 2002. Role of androgens in female genital sexual arousal: receptor expression, structure, and function. Fertil. Steril. 77, S11-S18.

Tuiten, A., Van Honk, J., Koppeschaar, H., Bernaards, C., Thijssen, J., Verbaten, R., 2000. Time course of effects of testosterone administration on sexual arousal in women. Arch. Gen. Psychiatry 57, 149-153.

van Anders, S.M., Hampson, E., 2005. Waist-to-hip ratio is positively associated with bioavailable testosterone but negatively associated with sexual desire in healthy premenopausal women. Psychosom. Med. 67, 246-250.

van Anders, S.M., Watson, N.V., 2006a. Menstrual cycle irregularities are associated with testosterone levels in healthy premenopausal women. Am. J. Hum. Biol. 18, 841-844.

van Anders, S.M., Watson, N.V., 2006b. Social neuroendocrinology: effects of social contexts and behaviours on sex steroids in humans. Hum. Nat. 17, 212-237.

van Anders, S.M., Chernick, A.B., Chernick, B.A., Hampson, E., Fisher, W.A., 2005. Preliminary clinical experience with androgen supplementation for pre- and post-menopausal women with hypoactive sexual desire. J. Sex Marital. Ther. 31, 1-13.

van Anders, S.M., Hampson, E., Watson, N.V., 2006. Seasonality, waist-tohip ratio, and salivary testosterone. Psychoneuroendocrinology 31, 895-899.

Wang, C., Plymate, S., Nieschlag, E., Paulsen, C.A., 1981. Salivary testosterone in men: further evidence of a direct correlation with free serum testosterone. J. Clin. Endocrinol. Metab. 53, 1021-1024.

Wood, R.I., 2004. Reinforcing aspects of androgens. Physiol. Behav. 83, 279-289. 